PROCEEDINGS OF THE

AMERICAN MATHEMATICAL SOCIETY

Volume 136, Number 8, August 2008, Pages 2729-2734

S 0002-9939(08)09274-5

Article electronically published on April 15, 2008

\title{
ON CONGRUENCES OF JACOBI FORMS
}

\author{
OLAV K. RICHTER
}

(Communicated by Ken Ono)

\begin{abstract}
We consider congruences and filtrations of Jacobi forms. More specifically, we extend Tate's theory of theta cycles to Jacobi forms, which allows us to prove a criterion for an analog of Atkin's $U$-operator applied to a Jacobi form to be nonzero modulo a prime.
\end{abstract}

\section{INTRODUCTION AND STATEMENT OF RESULTS}

The theory of modular forms modulo a prime $p$ has a long history and has been the source for many fruitful applications. Ono 10 gives a good overview, and he discusses several applications of congruences that involve Atkin's $U$-operator (see also Ahlgren and Ono [1, Elkies, Ono, and Yang [5], and Guerzhoy [6]). Chida and Kaneko 2 use Serre and Swinnerton-Dyer's theory of filtrations of modular forms modulo $p$ (see [13] and [15]) in combination with Tate's theory of theta cycles (see $\S 7$ of [7]) to establish a criterion for when the $p$-th coefficient of a modular form is not divisible by $p$. The purpose of this paper is to prove an analogous result for Jacobi forms of index 1 .

In [12], we study the action of the heat operator $L:=\frac{1}{(2 \pi i)^{2}}\left(8 \pi i \frac{\partial}{\partial \tau}-\frac{\partial^{2}}{\partial z^{2}}\right)$ on Jacobi forms of index 1. If $\phi$ is such a Jacobi form, then $L^{n}(\phi)$ (for all $n \in \mathbb{N}$ ) is a quasi-Jacobi form in the sense of Kawai and Yoshioka 9]. More precisely, there exist unique quasimodular forms $f$ and $g$ of weights $2 n+k-4$ and $2 n+k-6$, respectively, such that $L^{n}(\phi)=f E_{4,1}+g E_{6,1}$, where $E_{4,1}$ and $E_{6,1}$ are the Jacobi Eisenstein series of index 1 and weights 4 and 6 , respectively. For details on quasimodular forms, see Kaneko and Zagier [8]. We adopt the notation in [2]. Let $E_{k}(\tau):=1-\frac{2 k}{B_{k}} \sum_{n=1}^{\infty}\left(\sum_{d \mid n} d^{k-1}\right) q^{n}\left(q:=e^{2 \pi i \tau}, k \geq 2\right)$ denote the usual Eisenstein series. In particular, $E_{2}$ is the "quasimodular" Eisenstein series of weight 2. If $f \in \mathbb{C}\left[E_{2}, E_{4}, E_{6}\right]$ is quasimodular, then let $F(f ; X, Y, Z)$ be the polynomial in $X, Y$, and $Z$ such that $f(\tau)=F\left(f ; E_{2}(\tau), E_{4}(\tau), E_{6}(\tau)\right)$ and let $F^{(0)}:=F^{(0)}(f ; Y, Z):=F(f ; 0, Y, Z)$ be the "modular part". Note that if $p \geq 5$ is a prime, then the Hasse invariant $H_{p}(Y, Z):=F^{(0)}\left(E_{p-1} ; Y, Z\right)$ has $p$-integral rational coefficients.

Now we can state the main result of our paper.

Received by the editors June 25, 2007.

2000 Mathematics Subject Classification. Primary 11F50; Secondary 11F60.

(C)2008 American Mathematical Society 
Theorem 1. Let

$$
\phi(\tau, z)=\sum_{\substack{n, r \\ 4 n-r^{2} \geq 0}} c(n, r) q^{n} \zeta^{r} \in \mathbb{Z}[[q, \zeta]]
$$

be a Jacobi form of weight $k$ and index 1 , where $q:=e^{2 \pi i \tau}$ and $\zeta:=e^{2 \pi i z}(\tau \in \mathbb{H}$, $z \in \mathbb{C})$. Let $p>k$ be a prime such that $\phi \not \equiv 0(\bmod p)$, and let

$$
\phi(\tau, z) \mid U_{p}:=\sum_{\substack{n, r \\ 4 n-r^{2} \geq 0 \\ p \mid\left(4 n-r^{2}\right)}} c(n, r) q^{n} \zeta^{r}
$$

be the analog of Atkin's $U$-operator for Jacobi forms (see also [12]). If $p>2 k-5$, then $\phi \mid U_{p} \not \equiv 0(\bmod p)$. Suppose that $p<2 k-5$, and let us write $L^{\frac{3 p+3}{2}-k}(\phi)=$ $f E_{4,1}+g E_{6,1}$. Then the quasimodular forms $f$ and $g$ (and hence also $F^{(0)}$ and $G^{(0)}$ ) have $p$-integral rational coefficients and the following conditions are equivalent:

(1) $\phi \mid U_{p} \not \equiv 0(\bmod p)$,

(2) $H_{p}(Y, Z) \nmid F^{(0)}(\bmod p)$ or $\quad H_{p}(Y, Z) \nmid G^{(0)}(\bmod p)$.

In Section 2, we give a closed formula for the quasi-Jacobi form $L^{n}(\phi)$. In Section 3, we extend Tate's theory of theta cycles to Jacobi forms. More specifically, we establish a result on heat cycles of Jacobi forms which is a key ingredient in our proof of Theorem 1. Finally, in Section 4, we apply Theorem 1 to the explicit example $\phi_{10,1}:=\frac{1}{144}\left(E_{6} E_{4,1}-E_{4} E_{6,1}\right)$. We find that $\phi_{10,1} \mid U_{p} \equiv 0(\bmod p)$ for $p=5,11,13$, while $\phi_{10,1} \mid U_{p} \not \equiv 0(\bmod p)$ for $p=7$ and all primes $p>13$.

\section{JACOBI FORMS AND THE HEAT OPERATOR}

Let $\mathbb{Z}$ and $\mathbb{N}$ denote the sets of integers and nonnegative integers, respectively, and let $\mathbb{H} \subset \mathbb{C}$ be the complex upper half plane. We recall the definition of a Jacobi form (for more details, see Eichler and Zagier [4]).

Definition 1. A Jacobi form of weight $k$ and index $m(k, m \in \mathbb{N})$ is a holomorphic function $\phi: \mathbb{H} \times \mathbb{C} \rightarrow \mathbb{C}$ satisfying the transformation laws

$$
\phi\left(\frac{a \tau+b}{c \tau+d}, \frac{z}{c \tau+d}\right)=(c \tau+d)^{k} e^{2 \pi i m \frac{c z^{2}}{c \tau+d}} \phi(\tau, z), \text { for all }\left(\begin{array}{ll}
a & b \\
c & d
\end{array}\right) \in \mathrm{SL}_{2}(\mathbb{Z}),
$$

and

$$
\phi(\tau, z+\lambda \tau+\mu)=e^{-2 \pi i m\left(\lambda^{2} \tau+2 \lambda z\right)} \phi(\tau, z), \text { for all }(\lambda, \mu) \in \mathbb{Z}^{2} .
$$

Furthermore, one requires that a Jacobi form has a Fourier expansion of the form

$$
\phi(\tau, z)=\sum_{\substack{n, r \in \mathbb{Z} \\ 4 n m-r^{2} \geq 0}} c(n, r) q^{n} \zeta^{r} .
$$

We denote the vector space of Jacobi forms of weight $k$ and index $m$ by $J_{k, m}$.

In this paper we are solely interested in Jacobi forms of index 1. If $\phi \in J_{k, 1}$, then

$$
\mathcal{D} \phi:=L(\phi)-\frac{2 k-1}{6} \phi E_{2} \in J_{k+2,1}
$$


It is not important for our purposes, but $\mathcal{D} \phi$ can be explicitly characterized (for details, see [12]). For $\phi \in J_{k, 1}$, define the sequence $\phi_{r} \in J_{k+2 r, 1}$ recursively by

$$
\phi_{r+1}:=\mathcal{D} \phi_{r}-\frac{r\left(r+k-\frac{3}{2}\right)}{9} E_{4} \phi_{r-1}(r \geq 0)
$$

with initial condition $\phi_{0}=\phi$. Then the formula for $f_{r}$ on page 1272 of Choie and Eholzer [3] (with $\Phi=-\frac{1}{9} E_{4}$ and $\phi=\frac{1}{3} E_{2}$ ) yields the following closed formula.

Lemma 1. Let $\phi \in J_{k, 1}$. Then for all $n \in \mathbb{N}$, we have

$$
L^{n}(\phi)=n ! \sum_{j=0}^{n}\left(\begin{array}{c}
k-\frac{3}{2}+n \\
j
\end{array}\right) \frac{\phi_{n-j}}{(n-j) !}\left(\frac{E_{2}}{3}\right)^{j} .
$$

\section{Heat Cycles and the proof of Theorem 1}

We study heat cycles of Jacobi forms (generalizing Tate's theory of theta cycles), which allows us to prove Theorem 1. Let us briefly review congruences and filtrations of Jacobi forms. Throughout, let $p \geq 5$ be a prime. Set

$$
\widetilde{J}_{k, 1}:=\left\{\phi(\bmod p): \phi(\tau, z) \in J_{k, 1} \cap \mathbb{Z}[[q, \zeta]]\right\} .
$$

If $\phi \in J_{k, 1}$ has $p$-integral rational coefficients, then we denote its filtration modulo $p$ by

$$
\Omega(\phi):=\inf \left\{k: \phi(\bmod p) \in \widetilde{J}_{k, 1}\right\} .
$$

Recall the following facts:

Proposition 1 (Sofer [14]). Let $\phi(\tau, z) \in J_{k, 1} \cap \mathbb{Z}[[q, \zeta]]$ and $\psi(\tau, z) \in J_{k^{\prime}, 1} \cap \mathbb{Z}[[q, \zeta]]$ such that $0 \not \equiv \phi \equiv \psi(\bmod p)$. Then $k \equiv k^{\prime}(\bmod p-1)$.

Proposition $2([12])$. If $\phi(\tau, z) \in J_{k, 1} \cap \mathbb{Z}[[q, \zeta]]$, then $L(\phi)(\bmod p)$ is the reduction of a Jacobi form modulo $p$. Moreover, we have

$$
\Omega(L(\phi)) \leq \Omega(\phi)+p+1,
$$

with equality if and only if $p \nmid(2 \Omega(\phi)-1)$.

Propositions 1 and 2 play an important role in our following investigation of heat cycles of Jacobi forms.

Let $\phi(\tau, z) \in J_{k, 1} \cap \mathbb{Z}[[q, \zeta]]$ such that $\phi \not \equiv 0(\bmod p)$ and suppose that $\phi \mid U_{p} \equiv$ $0(\bmod p)$. Then $L^{p-1}(\phi) \equiv \phi(\bmod p)$. We use the terminology of $\S 7$ of $[7$ and we call $\phi_{1}$ a low point of its heat cycle if it occurs immediately after a fall, i.e., if $\phi_{1}=L^{A}(\phi)$ and $2 \Omega\left(L^{A-1} \phi\right) \equiv 1(\bmod p)$. Let $\phi_{1}$ be a low point of its heat cycle and let $c_{j}-1 \in \mathbb{N}$ be minimal such that

$$
2 \Omega\left(L^{c_{j}-1}\left(\phi_{1}\right)\right)=2\left(\Omega\left(\phi_{1}\right)+\left(c_{j}-1\right)(p+1)\right) \equiv 1(\bmod p),
$$

and let $b_{j} \in \mathbb{N}$ be defined by

$$
\Omega\left(L^{c_{j}}\left(\phi_{1}\right)\right)=\Omega\left(\phi_{1}\right)+c_{j}(p+1)-b_{j}(p-1) .
$$

An argument as in $\S 7$ of [7] shows that there is either one fall with $c_{1}=p-1$ and $b_{1}=p+1$, or there are two falls with $b_{1}=p-c_{2}$ and $b_{2}=p-c_{1}$. The first case occurs if and only if $2 \Omega\left(\phi_{1}\right) \equiv 5(\bmod p)$. In the second case, $\Omega\left(\phi_{1}\right)=a p+B$ with $1 \leq B \leq p$ and $p \neq 2 B-5$. If $p>2 B-5$, then we calculate that $c_{1}=\frac{p+3}{2}-B$, $c_{2}=\frac{p-5}{2}+B$, and $\Omega\left(L^{\frac{p+3}{2}-B}\left(\phi_{1}\right)\right)=a p-B+4$. If $p<2 B-5$, then we obtain 
that $c_{1}=\frac{3 p+3}{2}-B, c_{2}=-\frac{p+5}{2}+B$, and $\Omega\left(L^{\frac{3 p+3}{2}-B}\left(\phi_{1}\right)\right)=(a+2) p-B+4$. In particular, if $a=0$, then $B \geq 4$ and we necessarily have that $p<2 B-5$.

On the other hand, if $\phi(\tau, z)=\sum c(n, r) q^{n} \zeta^{r} \in J_{k, 1} \cap \mathbb{Z}[[q, \zeta]]$ such that $\Omega(\phi)=$ $k<p<2 k-5$ and $\phi \mid U_{p} \not \equiv 0(\bmod p)$, then $L(\phi)$ must be a low point of its heat cycle. If it were not, then $\psi(\tau, z):=\sum_{p \nmid\left(4 n-r^{2}\right)} c(n, r) q^{n} \zeta^{r}$ would satisfy $\psi \mid U_{p} \equiv$ $0(\bmod p), L(\psi) \equiv L(\phi)(\bmod p)$, and $\Omega(\psi)=\Omega(\phi)$. Then $\Omega(\psi-\phi)=\Omega(\phi)$ and the fact that $2 \Omega(\phi) \not \equiv 1(\bmod p)$ would imply that

$$
\Omega(L(\psi-\phi)) \underset{(2)}{=} \Omega(\psi-\phi)+p+1=\Omega(\phi)+p+1,
$$

which is a contradiction, since $L(\psi-\phi) \equiv 0(\bmod p)$. We conclude that $L(\phi)$ is a low point of its heat cycle and we find that $\Omega\left(L^{\frac{3 p+3}{2}-k}(\phi)\right)=3 p-k+3$.

We summarize our investigation of heat cycles:

Proposition 3. Let $\phi(\tau, z) \in J_{k, 1} \cap \mathbb{Z}[[q, \zeta]]$ such that $\phi \not \equiv 0(\bmod p)$. If $p>2 k-5$, then $\phi \mid U_{p} \not \equiv 0(\bmod p)$. If $k<p<2 k-5$, then

$$
\Omega\left(L^{\frac{3 p+3}{2}-k}(\phi)\right)= \begin{cases}3 p-k+3 & \text { if } \phi \mid U_{p} \not \equiv 0(\bmod p), \\ 2 p-k+4 & \text { if } \phi \mid U_{p} \equiv 0(\bmod p) .\end{cases}
$$

Note that $E_{4,1}$ and $E_{6,1}$ form a basis (over the ring of modular forms) of $J_{k, 1}$. It is easy to see that $E_{4,1}$ and $E_{6,1}$ are relatively prime modulo $p$, and hence Lemma 5 of [15] immediately implies the following result.

Lemma 2. If $\phi=f E_{4,1}+g E_{6,1} \in J_{k, 1}$ has integral coefficients, then the following conditions hold:

(1) If $\Omega(\phi)=k$, then $H_{p}(Y, Z) \nmid F^{(0)}(\bmod p)$ or $H_{p}(Y, Z) \nmid G^{(0)}(\bmod p)$.

(2) If $\Omega(\phi)<k$, then $H_{p}(Y, Z) \mid F^{(0)}(\bmod p)$ and $H_{p}(Y, Z) \mid G^{(0)}(\bmod p)$.

Now we are in a position to prove Theorem [1, Let $\phi(\tau, z) \in J_{k, 1} \cap \mathbb{Z}[[q, \zeta]]$ such that $k<p<2 k-5$. Then Lemma 1 with $n=\frac{3 p+3}{2}-k$ shows that

$$
L^{\frac{3 p+3}{2}-k}(\phi)=f E_{4,1}+g E_{6,1} \equiv F^{(0)} E_{4,1}+G^{(0)} E_{6,1}(\bmod p),
$$

i.e., $L^{\frac{3 p+3}{2}-k}(\phi)(\bmod p) \in \widetilde{J}_{3 p-k+3,1}$, and Theorem 1 follows from Proposition 3 and Lemma 2 .

\section{An EXAMPLE}

We illuminate Theorem 1 and Proposition 3 with a concrete example. Let $\phi_{10,1}=\frac{1}{144}\left(E_{6} E_{4,1}-E_{4} E_{6,1}\right)$ be the unique (up to a scalar) Jacobi cusp form of weight 10 and index 1. Recall Ramanujan's [1] identities and their Jacobi form generalizations in [12]:

$$
\begin{aligned}
L\left(E_{2}\right) & =4 \Theta\left(E_{2}\right)=\frac{1}{3}\left(E_{2}^{2}-E_{4}\right), \\
L\left(E_{4}\right) & =4 \Theta\left(E_{4}\right)=\frac{4}{3}\left(E_{4} E_{2}-E_{6}\right), \\
L\left(E_{6}\right) & =4 \Theta\left(E_{6}\right)=2\left(E_{6} E_{2}-E_{4}^{2}\right), \\
L\left(E_{4,1}\right) & =\frac{7}{6}\left(E_{4,1} E_{2}-E_{6,1}\right), \\
L\left(E_{6,1}\right) & =\frac{11}{6}\left(E_{6,1} E_{2}-E_{4} E_{4,1}\right),
\end{aligned}
$$


where $\Theta:=q \frac{d}{d q}=\frac{1}{2 \pi i} \frac{d}{d \tau}$ is the Ramanujan theta operator. If $p=11$, then $E_{4} E_{6}=E_{10} \equiv 1(\bmod 11)$ and a direct computation (with Mathematica) using the formulas in (3) reveals that

$$
\begin{aligned}
L^{8}\left(\phi_{10,1}\right) & \equiv E_{4} E_{6}\left(3 E_{4}^{3}+2 E_{6}^{2}\right) E_{4,1}+6\left(E_{4} E_{6}\right)^{2} E_{6,1} \\
& \equiv\left(3 E_{4}^{3}+2 E_{6}^{2}\right) E_{4,1}+\left(6 E_{10}\right) E_{6,1} \quad(\bmod 11) .
\end{aligned}
$$

Hence $\Omega\left(L^{8}\left(\phi_{10,1}\right)\right)=16$ and $\phi_{10,1} \mid U_{11} \equiv 0(\bmod 11)$. Similarly if $p=13$, then $6 E_{4}^{3}+8 E_{6}^{2} \equiv E_{12} \equiv 1(\bmod 13)$, and we find that

$$
\begin{aligned}
L^{11}\left(\phi_{10,1}\right) & \equiv 9 E_{4}\left(6 E_{4}^{3}+8 E_{6}^{2}\right)^{2} E_{4,1}+\left(6 E_{4}^{3}+8 E_{6}^{2}\right)\left(4 E_{4}^{2} E_{6}\right) E_{6,1} \\
& \equiv\left(9 E_{4} E_{12}\right) E_{4,1}+\left(4 E_{4}^{2} E_{6}\right) E_{6,1} \quad(\bmod 13) .
\end{aligned}
$$

Hence $\Omega\left(L^{11}\left(\phi_{10,1}\right)\right)=20$ and $\phi_{10,1} \mid U_{13} \equiv 0(\bmod 13)$. Furthermore, Proposition 3 implies that $\phi_{10,1} \mid U_{p} \not \equiv 0(\bmod p)$ for all primes $p>13$.

Note that Theorem 1 and Proposition 3 provide no information on primes $p<11$. Nevertheless, the table of coefficients of Jacobi forms of index 1 on p. 141 of [4] shows that $\phi_{10,1} \mid U_{7} \not \equiv 0(\bmod 7)$. Finally, if $p=5$, then we calculate that $\Omega\left(L\left(\phi_{10,1}\right)\right)=16, \Omega\left(L^{2}\left(\phi_{10,1}\right)\right)=22, \Omega\left(L^{3}\left(\phi_{10,1}\right)\right)=28$, and $\Omega\left(L^{4}\left(\phi_{10,1}\right)\right)=\Omega\left(\phi_{10,1}\right)=10$. If $\phi_{10,1} \mid U_{5} \not \equiv 0(\bmod 5)$, then (with the same argument as before) $\Omega\left(L\left(\phi_{10,1}\right)\right)$ would be a low point of its heat cycle, which is obviously not the case. Hence $\phi_{10,1} \mid U_{5} \equiv 0(\bmod 5)$.

We conclude that $\phi_{10,1} \mid U_{p} \equiv 0(\bmod p)$ for $p=5,11,13$, while $\phi_{10,1} \mid U_{p} \not \equiv$ $0(\bmod p)$ for $p=7$ and all primes $p>13$.

\section{ACKNOWLEDGMENT}

The author thanks Anne Shepler for her help with Mathematica.

\section{REFERENCES}

1. S. Ahlgren and K. Ono, Arithmetic of singular moduli and class polynomials, Compos. Math. 141 (2005), no. 2, 293-312. MR2134268 (2006a:11058)

2. M. Chida and M. Kaneko, On ordinary primes for modular forms and the theta operator, Proc. Amer. Math. Soc. 135 (2007), no. 4, 1001-1005. MR2262900

3. Y. Choie and W. Eholzer, Jacobi forms and generalized RC-algebras, Rocky Mountain J. Math. 31 (2001), no. 4, 1265-1275. MR.1895295(2003d:11072)

4. M. Eichler and D. Zagier, The theory of Jacobi forms, Birkhäuser, Boston, 1985. MR781735 (86j:11043)

5. N. Elkies, K. Ono, and T. Yang, Reduction of CM elliptic curves and modular function congruences, Internat. Math. Res. Notices 2005, no. 44, 2695-2707. MR2181309 (2006k:11076)

6. P. Guerzhoy, On U(p)-congruences, Proc. Amer. Math. Soc. 135 (2007), no. 9, 2743-2746. MR 2317947

7. N. Jochnowitz, A study of the local components of the Hecke algebra mod l, Trans. Amer. Math. Soc. 270 (1982), no. 1, 253-267. MR642340 (83e:10033a)

8. M. Kaneko and D. Zagier, A generalized Jacobi theta function and quasimodular forms, in: The moduli space of curves (Texel Island, 1994), Progr. Math. 129, pp. 165-172, Birkhäuser, Boston, 1995. MR 1363056 (96m:11030)

9. T. Kawai and K. Yoshioka, String partition functions and infinite products, Adv. Theor. Math. Phys. 4 (2000), no. 2, 397-485. MR.1838446 (2002g:11054)

10. K. Ono, The web of modularity: Arithmetic of the coefficients of modular forms and q-series, CBMS Regional Conference Series in Mathematics, vol. 102, Published for the Conference Board of the Mathematical Sciences, Washington, DC, by the Amer. Math. Soc., Providence, RI, 2004. MR2020489 (2005c:11053) 
11. S. Ramanujan, On certain arithmetical functions, Trans. Camb. Phil. Soc. 22 (1916), 159-184 (Collected Papers, No. 18).

12. O. Richter, The action of the heat operator on Jacobi forms, to appear in the Proceedings of the AMS.

13. J-P. Serre, Formes modulaires et fonctions zeta p-adiques, in: Modular functions of one variable III, Lecture Notes in Math. 350, pp. 191-268, Springer, 1973. MR0404145 (53:7949a)

14. A. Sofer, p-adic aspects of Jacobi forms, J. Number Theory 63 (1997), no. 2, 191-202. MR 1443756 (98b:11058)

15. H. P. F. Swinnerton-Dyer, On l-adic representations and congruences for coefficients of modular forms, in: Modular functions of one variable III, Lecture Notes in Math. 350, pp. 1-55, Springer, 1973. MR0406931(53:10717a)

Department of Mathematics, University of North Texas, Denton, Texas 76203

E-mail address: richter@unt.edu 